\title{
A Multicast Approach for Constructive Interference Precoding in MISO Downlink Channel
}

\author{
Maha Alodeh Symeon Chatzinotas Björn Ottersten \\ SnT-Interdisciplinary Centre for Security, Reliability and Trust, University of Luxembourg \\ 4, rue Alphonse Weicker, L-2721 Luxembourg \\ e-mail:\{maha.alodeh, symeon.chatzinotas, bjorn.ottersten\}@uni.lu
}

\begin{abstract}
This paper studies the concept of jointly utilizing the data information (DI) and channel state information (CSI) in order to design symbol-level precoders for a multiple input and single output (MISO) downlink channel. In this direction, the interference among the simultaneous data streams is transformed to useful signal that can improve the signal to interference noise ratio (SINR) of the downlink transmissions. We propose a maximum ratio transmissions (MRT) based algorithm that jointly exploits DI and CSI to gain the benefits from these useful signals. In this context, a novel framework to minimize the power consumption is proposed by formalizing the duality between the constructive interference downlink channel and the multicast channels. The numerical results have shown that the proposed schemes outperform other state of the art techniques. []
\end{abstract}

\section{INTRODUCTION}

In the last decade, multiuser MISO techniques have attracted a lot of attention due to their capability of serving co-channel users in the same frequency and time slots. The applications of these techniques vary according to the requested service. The first service type is known as broadcast in which a transmitter has a common message to be sent to multiple receivers. In physical layer research, this service has been studied under the term of multicasting [1]. Since a single data stream is sent to all receivers, there is no need to combat the interuser interference. In the remainder of this paper, this case will be referred to as multicast. The second service type is known as unicast, in which a transmitter has an individual message for a receiver. Due to the nature of the wireless medium and the use of multiple antennas, multiple simultaneous unicast transmissions are possible in the downlink of a base station (BS). In these cases, multiple streams are simultaneously sent, which motivates precoding techniques that mitigate the interuser interference. In information theory terms, this service type has been studied using the broadcast channel [2]. In the remainder of this paper, this case will be referred to as downlink.

The concept of jointly using the DI and CSI in symbol level was originally proposed in [6]- [7]. Taking into account the data information, the cross correlations among the users' subspaces can be optimized. The contributions of this paper can be summarized in the following points:

This work was supported by the National Research Fund (FNR) of Luxembourg under the AFR grant (reference 4919957) for the project Smart Resource Allocation Techniques for Satellite Cognitive Radio.
- As preliminary step, we develop a new constructive interference algorithm, called constructive interference maximum ratio transmissions (CIMRT). This technique is shown to outperform the constructive rotation zero forcing precoding (CRZF) in [7].

- We illustrate the relation between the constructive interference precoding and constrained constellation multicast precoding and as a result we establish a suitable upperbound for constructive interference precoding systems. To characterize the multicast performance, we derive the minimum power that is required to satisfy the quality of service of multicast MISO for MPSK inputs.

- We verify the convexity of the power minimization with the additional constraints. Therefore, we formulate the optimal precoders for power minimization for constrained constellation multicast channel. Finally, we show that there is a duality between constructive interference downlink channel (CIDC) and constrained constellation multicast channel (CCMC). Based on this duality, we derive the optimal precoding for constructive interference downlink channel.

Notation: We use boldface upper and lower case letters for matrices and column vectors, respectively. $(\cdot)^{H},(\cdot)^{*}$ stand for Hermitian transpose and conjugate of $(\cdot)$. $\mathbb{E}(\cdot)$ and $\|\cdot\|$ denote the statistical expectation and the Euclidean norm, $\otimes$ denotes the kronecker product, and $\mathbf{A} \succeq \mathbf{0}$ is used to indicate the positive semidefinite matrix. $\angle(\cdot),|\cdot|$ are the angle and magnitude of $(\cdot)$ respectively. $\mathcal{R}(\cdot), \mathcal{I}(\cdot)$ are the real and the imaginary part of $(\cdot)$. Finally, the vector of all zeros with length of $K$ is defined as $\mathbf{0}^{K \times 1}$.

\section{System AND Signal Models}

We consider a single-cell multiple-antenna downlink scenario, where a single BS is equipped with $n t$ transmit antennas that supports data traffic to $K$ user terminals, each one of them is equipped with a single receiving antenna. We assume a quasi static block fading channel $\mathbf{h}_{j} \in \mathbb{C}^{1 \times n t}$ between the BS antennas and the $j^{t h}$ user. Let $\mathbf{w}_{k}$ be the $\mathbb{C}^{n t \times 1}$ normalized precoding vector for the user $j$. The received signal at $j^{t h}$ user $y_{j}$ is given by

$$
y_{j}=\sqrt{p_{j}} \mathbf{h}_{j} \mathbf{w}_{j} d_{j}+\sum_{k \neq j} \sqrt{p_{k}} \mathbf{h}_{j} \mathbf{w}_{k} d_{k}+z_{j}
$$

where $z_{j}$ denotes the noise at $j^{\text {th }}$ receiver, which is assumed independent and identically distributed (i.d.d) complex Gaus- 
sian distributed variable $\mathcal{C N}(0,1)$. A more compact system formulation is obtained by stacking the received signals and the noise components for the set of $\mathrm{K}$ selected users as $\mathbf{y}=\mathbf{H W P}^{\frac{1}{2}} \mathbf{d}+\mathbf{z}$, with $\mathbf{H}=\left[\mathbf{h}_{1}, \ldots, \mathbf{h}_{K}\right]^{T} \in \mathbb{C}^{K \times n t}$, $\mathbf{W}=\left[\mathbf{w}_{1}, \ldots, \mathbf{w}_{K}\right] \in \mathbb{C}^{n t \times K}$ as the compound channel and precoding matrices. Notice that the transmitted signal $\mathbf{d} \in \mathbb{C}^{K \times 1}$ includes the uncorrelated data symbols $d_{k}(t)$ for all users with $\mathbb{E}\left[\left|d_{j}\right|^{2}\right]=1, \mathbf{P}^{\frac{1}{2}}$ is the power allocation matrix $\mathbf{P}^{\frac{1}{2}}=\operatorname{diag}\left(\sqrt{p}_{1}, \ldots, \sqrt{p}_{K}\right)$. The CSI and DI are available at the transmitter side.

\section{Constructive Interference Precoding}

The interference among the simultaneous spatial streams leads to deviation of the received symbols from their detection region. However, in some cases (e.g. M-PSK) this interference pushes the received symbols further into the correct detection region and, as a consequence it enhances the system performance. Therefore, the interference can be classified into constructive or destructive based on whether it facilitates or deteriorates the correct detection of the received symbol.

\section{A. Constructive Interference Definition}

Assuming both DI and CSI is available at the transmitter, the normalized created interference from the $k^{\text {th }}$ data stream on $j^{\text {th }}$ user can be formulated as:

$$
\psi_{j k}=\frac{\mathbf{h}_{j} \mathbf{w}_{k}}{\left\|\mathbf{h}_{j}\right\|\left\|\mathbf{w}_{k}\right\|} .
$$

Since the adopted modulations are M-PSK ones, a definition for constructive interference can be stated as

Lemma 1: For any M-PSK modulated symbol $d_{k}$ is said to receive constructive interference from another simultaneously transmitted symbol $d_{j}$ which is associated with $\mathbf{w}_{j}$ if and only if the following inequalities hold

$$
\begin{aligned}
& \angle d_{j}-\frac{\pi}{M} \leq \tan ^{-1}\left(\frac{\mathcal{I}\left\{\psi_{j k} d_{k}\right\}}{\mathcal{R}\left\{\psi_{j k} d_{k}\right\}}\right) \leq \angle d_{j}+\frac{\pi}{M} \\
& \mathcal{R}\left\{d_{k}\right\} . \mathcal{R}\left\{\psi_{k j} d_{j}\right\}>0, \mathcal{I}\left\{d_{k}\right\} . \mathcal{I}\left\{\psi_{k j} d_{j}\right\}>0 .
\end{aligned}
$$

Proof: For any M-PSK modulated symbol, the region of correct detection lies in $\phi_{j} \in\left[\angle d_{j}-\frac{\pi}{M}, \angle d_{j}+\frac{\pi}{M}\right]$. In order for the interference to be constructive, the received interfering signal should lie in the region of the target symbol. For the first condition, the $\arctan (\cdot)$ function checks whether the received interfering signal originating from the $d_{k}{ }^{t h}$ transmit symbol is located in the detection region of the target symbol. However, the trigonometric functions are not one-to-one functions. This means that it manages to check the two quadrants which the interfering symbol may lie in. To find which one of these quadrants is the correct one, an additional constraint is added to check the sign compatibility of the target and received interfering signals.

\section{Constructive Interference Precoding For MISO DOWNLINK CHANNELS}

In the remainder of this paper, it is assumed that the transmitter is capable of designing precoding on symbol level for each time instance utilizing both CSI and DI, so that the created interference among the simultaneous spatial streams is constructive.

\section{A. Correlation Rotation Zero Forcing Precoding (CRZF)}

The precoder aims at minimizing the mean square error while it takes into the account the rotated constructive interference [7]. The optimization problem can be formulated as

$$
\mathcal{J}=\min _{\mathbf{W}} \quad \mathbb{E}\left\{\left\|\mathbf{R}_{\phi} \mathbf{d}-(\mathbf{H W d}+\mathbf{z})\right\|^{2}\right\} .
$$

The solution can be easily expressed as

$$
\mathbf{W}_{C R Z F}=\gamma \mathbf{H}^{H}\left(\mathbf{H H}^{H}\right)^{-1} \mathbf{R}_{\phi}
$$

where $\gamma=\sqrt{\frac{P}{\operatorname{tr}\left(\mathbf{R}_{\phi}^{H}\left(\mathbf{H H}^{H}\right)^{-1} \mathbf{R}_{\phi}\right)}}$ ensures the power normalization. The cross correlation factor between the $j^{\text {th }}$ user's channel and transmitted $k^{\text {th }}$ data stream can be expressed as

$$
\rho_{j k}=\frac{\mathbf{h}_{j} \mathbf{h}_{k}^{H}}{\left\|\mathbf{h}_{k}\right\|\left\|\mathbf{h}_{j}\right\|} .
$$

The relative phase $\phi_{i j}$ that grants the constructive simultaneous transmissions can be expressed as

$$
\phi_{i j}=\angle d_{j}-\angle\left(\rho_{i j} \cdot d_{i}\right) .
$$

The corresponding rotation matrix can be implemented as:

$$
\mathbf{R}_{\phi}(j, k)=\rho_{j k} \exp \left(\phi_{j k} i\right),
$$

and the received signal at $j^{\text {th }}$ user can be expressed as

$$
y_{j} \stackrel{a}{=} \gamma\left\|\mathbf{h}_{j}\right\|\left(\sum_{k=1}^{K} \rho_{j k} d_{k}\right) \stackrel{b}{=} \gamma\left\|\mathbf{h}_{j}\right\|\left(\sum_{k=1}^{K} \varepsilon_{j k}\right) d,
$$

where $\varepsilon_{j k}$ has the same magnitude as $\rho_{j k}$ but with different phase, and $d: d \in \mathbb{C}^{1 \times 1},|d|=1, \angle d=\theta, \theta \in[0,2 \pi]$. By taking a look at (9-b), it has a multicast formulation since it seems for each user that BS sends the same symbol for all users.

Remark 1: It can be noted that this solution includes a zero forcing step and a correlation step $\mathbf{R}_{\phi}$. The correlation step aims at making the transmit signals constructively received at each user. Unfortunately, this design fails when we deal with co-linear users $\rho_{j k} \rightarrow 1$. However, intuitively having co-linear users should create more constructive interference and higher gain should be anticipated. It can be easily concluded that the source of this contradiction is the zero forcing step. In an effort to overcome the problem, we propose a new precoding technique in the next section. 


\section{B. Proposed Constructive Interference Maximum Ratio Trans- mission (CIMRT)}

The maximum ratio transmissions (MRT) are not suitable for simultaneous downlink transmissions in MISO system due to the intolerable amount of the created interference. On the other hand, this feature makes it a good candidate for constructive interference. The naive maximum ratio transmission (nMRT) can be formulated as

$$
\mathbf{W}_{\mathrm{nMRT}}=\left[\frac{\mathbf{h}_{1}{ }^{H}}{\left\|\mathbf{h}_{1}\right\|}, \frac{\mathbf{h}_{2}{ }^{H}}{\left\|\mathbf{h}_{2}\right\|}, \ldots, \frac{\mathbf{h}_{K}{ }^{H}}{\left\|\mathbf{h}_{K}\right\|}\right] .
$$

A new look at the received signal can be viewed by exploiting the singular value decomposition of $\mathbf{H}=\mathbf{S V D}$, and $\mathbf{W}_{M R T}=\mathbf{D}^{H} \mathbf{V}^{\prime} \mathbf{S}^{H}$ as follows

$$
\mathbf{y}=\mathbf{H W d}=\mathbf{S V D D}^{H} \mathbf{V}^{\prime} \mathbf{S}^{H} \mathbf{P}^{1 / 2} \mathbf{d}
$$

$$
\mathbf{G}=\mathbf{S V V}^{\prime}, \quad \mathbf{B}=\mathbf{S}^{H}
$$

where $\mathbf{S} \in \mathbb{C}^{K \times K}$ is a unitary matrix that contains the leftsingular vectors of $\mathbf{H}$, the matrix $\mathbf{V}$ is an $K \times n t$ diagonal matrix with nonnegative real numbers on the diagonal, and $\mathbf{D} \in \mathbb{C}^{n t \times n t}$ contains right-singular vectors of $\mathbf{H}$. $\mathbf{V}^{\prime}$ is the power scaled of $\mathbf{V}$ to normalize each column in $\mathbf{W}_{M R T}$ to unit. The received signal can be as

$$
y_{j}=\left\|\mathbf{h}_{j}\right\|\left(\sum_{k=1}^{K} \sqrt{p_{k}} \rho_{j k} d_{k}\right) .
$$

Utilizing the reformulation of $\mathbf{y}$ in $(11)$, the received signal can be written as

$$
y_{j}=\left\|\mathbf{g}_{j}\right\| \sum_{k=1}^{K} \sqrt{p_{k}} \xi_{j k} d_{k}=\left\|\mathbf{g}_{j}\right\| \sum_{k=1}^{K} \sqrt{p_{k}} \xi_{j k} \exp \left(\theta_{k}\right) d
$$

where $\mathbf{g}_{j}$ is the $j^{\text {th }}$ row of the matrix $\mathbf{G}, \xi_{j k}=\frac{\mathbf{g}_{j} \mathbf{b}_{k}}{\left\|\mathbf{g}_{i}\right\|}$. Since $\mathbf{B}$ is a unitary matrix, it can have uncoupled rotations which can grant the constructivity of interference. Let $\mathbf{R}_{k j}$ be the rotation matrix in the $\left(\mathbf{b}_{k}, \mathbf{b}_{j}\right)$-plane, which performs an orthogonal rotation of the $k^{t h}$ and $j^{\text {th }}$ columns of a unitary matrix while keeping the others fixed, thus preserving unitarity. Assume without loss of generality that $k>j$. The rotation matrix in the $\left(\mathbf{b}_{k}, \mathbf{b}_{j}\right)$-plane can be written as

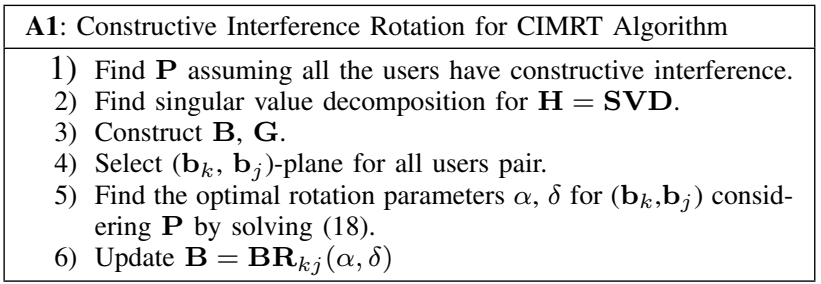

where the non trivial entries appear at the intersections of $k^{\text {th }}$ and $j^{\text {th }}$ rows and columns. Hence, any unitary matrix $\mathbf{B}^{\prime}$ can be expressed using the following parameterization

$$
\mathbf{B}^{\prime}=\mathbf{B} \prod_{j=1}^{K} \prod_{k=j+1}^{K} \mathbf{R}_{k j} \text {. }
$$

It can be seen from the structure of the matrix in 15 that rotation in the $\left(\mathbf{b}_{k}, \mathbf{b}_{j}\right)$-plane does not change the directions of the remaining beamforming vectors. Therefore, it just modifies the value of $\xi_{k k}$, and the precoder reads as

$$
\mathbf{W}_{C I M R T}=\mathbf{D}^{H} \mathbf{V}^{\prime} \mathbf{B}^{\prime} \text {. }
$$

To grant constructive interference, we need to rotate the $\left(\mathbf{b}_{k}, \mathbf{b}_{j}\right)$-plane by formulating the rotation as a set of non-linear equations as

$$
\begin{aligned}
\xi_{k k}^{\prime} d_{k} & =\xi_{k k} \cos (\alpha) d_{k}-\xi_{k j} \sin (\alpha) e^{-j \delta} d_{j} \\
\xi_{j j}^{\prime} d_{j} & =\xi_{j k} \sin (\alpha) e^{j \delta} d_{k}+\xi_{j j} \cos (\alpha) d_{j}
\end{aligned}
$$

Since the set of non-linear equations can have different roots, the function needs to be evaluated at the obtained root in order to find the optimal ones. The optimal solution can be found when solving for $\xi_{k k}^{\prime}=\sqrt{\xi_{k k}^{2}+\xi_{k j}^{2}}, \xi_{j j}^{\prime}=\sqrt{\xi_{j j}^{2}+\xi_{j k}^{2}}$. Sometimes it is not feasible to solve for $\xi_{k k}^{*}$ and $\xi_{j j}^{*}$, and their values need to be reduced correspondingly. The proposed algorithm can be illustrated in the following table:

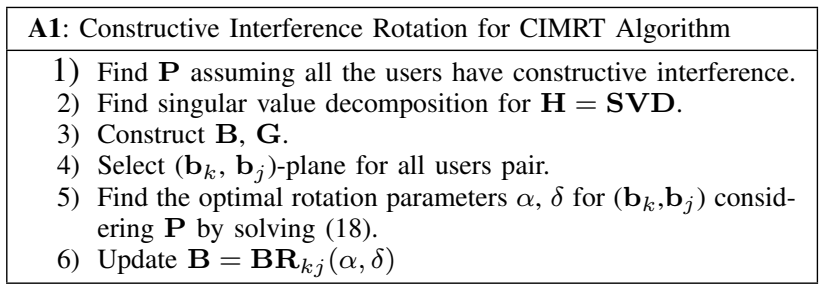

\section{Multicast MisO Systems}

The multicast channel is defined as the channel in which a multiantenna transmitter sends a single message to multiple single antenna users [1]- [5].

\section{A. Constrained M-PSK Multicast Transmissions}

The optimal input covariance for power minimization in multicast system can be found as a solution of the following optimization

$$
\min _{\mathbf{Q}: \mathbf{Q} \succeq 0} \quad \operatorname{tr}(\mathbf{Q}) \quad \text { s.t. } \quad \mathbf{h}_{j} \mathbf{Q} \mathbf{h}_{j}^{H} \geq \zeta_{j} \quad, \forall j \in K .(19)
$$

where $\zeta_{j}$ is the required SNR threshold for $j^{t h}$ user. Different solutions in the literature were proposed as in [1]. However, for M-PSK inputs we should design the multicast precoders so that the received signal falls into the detection region of desired symbol. Assuming a unit-rank solution for $\mathbf{Q}$, the optimization problem can be written as follows

$$
\begin{array}{cll}
\mathbf{w}_{\mathrm{CMC}}(d, \mathbf{H})=\arg \min _{\mathbf{w}} & \operatorname{tr}\left(\mathbf{w} \mathbf{w}^{H}\right) & \\
\text { s.t. } & \angle\left(\mathbf{h}_{j} \mathbf{w}\right)=\angle(d) & \forall j \in K \\
& \mathbf{h}_{j} \mathbf{w} \mathbf{w}^{H} \mathbf{h}_{j}^{H} \geq \zeta_{j} & \forall j \in K .(20)
\end{array}
$$

Here, we have $2 K$ additional constraints which limit the performance of (20) in comparison to (19). These constraints grant the reception of the symbol $d$ with the target SNR $\zeta_{j}$ for the $j^{t h}$ user. More flexible constraints for the detection region of the target symbols are discussed in [8]. The minimum transmit power in (19)-20) occurs when the inequality constraints are replaced by equality (i.e. all users should achieve their target threshold SNR). We can reformulate the constraint as 


$$
\begin{array}{lll}
\min _{\mathbf{w}} & \left(\mathbf{w}^{H} \mathbf{w}\right) & \\
\text { s.t. } & \mathcal{I}\left\{\mathbf{h}_{j} \mathbf{w}\right\}=\sqrt{\zeta_{j}} \mathcal{I}\{d\} \quad, \forall j \in K \\
& \mathcal{R}\left\{\mathbf{h}_{j} \mathbf{w}\right\}=\sqrt{\zeta_{j}} \mathcal{R}\{d\} \quad, \forall j \in K
\end{array}
$$

A final formulation can expressed as

$$
\begin{array}{ll}
\min _{\mathbf{w}} & \left(\mathbf{w}^{H} \mathbf{w}\right) \\
\text { s.t. } & \frac{\mathbf{h}_{j} \mathbf{w}-\left(\mathbf{h}_{j} \mathbf{w}\right)^{H}}{2 i}=\sqrt{\zeta_{j}} \mathcal{I}\{d\}, \forall j \in K \\
& \frac{\mathbf{h}_{j} \mathbf{w}+\left(\mathbf{h}_{j} \mathbf{w}\right)^{H}}{2}=\sqrt{\zeta_{j}} \mathcal{R}\{d\}, \forall j \in K .
\end{array}
$$

It can be viewed that the constraints in (20) are turned from inequality constraints to equality constraint (21)-(22) due to signal aligning requirements. The Lagrangian function can be derived as follows

$$
\begin{aligned}
\mathcal{L}(\mathbf{w}) & =\mathbf{w}^{H} \mathbf{w}+\sum_{j} \mu_{j}\left(-0.5 i\left(\mathbf{h}_{j} \mathbf{w}-\mathbf{w}^{H} \mathbf{h}_{j}^{H}\right)-\sqrt{\zeta_{t h}^{i}} \mathcal{I}\{d\}\right) \\
& +\sum_{j} \alpha_{j}\left(\left(\mathbf{h}_{j} \mathbf{w}+\mathbf{w}^{H} \mathbf{h}_{j}^{H}\right)-\sqrt{\zeta_{t h}^{i}} \mathcal{R}\{d\}\right)
\end{aligned}
$$

where $\mu_{j}$ and $\alpha_{j}$ are the Lagrangian dual variables. The derivative for the Lagrangian function can be written as

$$
\frac{d \mathcal{L}(\mathbf{w})}{d \mathbf{w}^{*}}=\mathbf{w}+0.5 i \sum_{j} \mu_{j} \mathbf{h}_{j}^{H}+0.5 \sum_{j} \alpha_{j} \mathbf{h}_{j}^{H}
$$

By equating this term to zero, w can be written as

$$
\mathbf{w}_{C M C}=-0.5 i \sum_{j=1}^{K} \mu_{j} \mathbf{h}_{j}^{H}-0.5 \sum_{j} \alpha_{j} \mathbf{h}_{j}^{H} \equiv \sum_{j=1}^{K} \nu_{j} \mathbf{h}_{j}^{H}
$$

where $\nu_{j} \in \mathbb{C}=-0.5 i \mu_{j}-0.5 \alpha_{j}$. The optimal values of the Lagrangian variables $\mu_{j}$ and $\alpha_{j}$ can be found by substituting $\mathbf{w}$ in the constraints (22) which result in solving the simultaneous set of $2 K$ equations 26. The final constrained constellation multicast precoder can be found by substituting all $\mu_{j}$ and $\alpha_{j}$ in (25).

Corollary 1: The optimal precoding for power minimization $\mathbf{w}_{C M C}$ in CCMC must span the subspaces of each user's channel.

Using 25, we can rewrite the received signal at $j^{\text {th }}$ receiver as

$$
\begin{aligned}
y_{j} & =\mathbf{h}_{j} \mathbf{w}_{C M C} d+z_{j}=\mathbf{h}_{j} \sum_{k=1}^{K} \nu_{k} \mathbf{h}_{k}^{H} d+z_{j} \\
& =\left\|\mathbf{h}_{j}\right\| \sum_{k=1}^{K}\left\|\mathbf{h}_{k}\right\| \nu_{k} \rho_{j k} d+z_{j} \\
& \equiv \mathbf{h}_{j}\left[\begin{array}{lll}
\left|\nu_{1}\right| * \mathbf{h}_{1}^{H} & \ldots & \left|\nu_{K}\right| * \mathbf{h}_{K}^{H}
\end{array}\right]\left[\begin{array}{c}
d * 1 \angle\left(\nu_{1}\right) \\
\vdots \\
d * 1 \angle\left(\nu_{K}\right)
\end{array}\right]+z_{j} .
\end{aligned}
$$

From 27, the constellation constrained multicast can be formulated as a constructive interference downlink channel with set of precoders $\mathbf{h}_{1}^{H}, \ldots, \mathbf{h}_{K}^{H}$, each one of these precoder is allocated with power $\left|\nu_{k}\right|$ and associated with symbol $d * 1 \angle \nu_{k}$.

\section{B. From Multicast to Constructive Interference}

In multicast, the cross correlations among users' channel are exploited to aid the transmission of the data symbols. The same cross correlations are translated to interference in the downlink channel due to the individuality of each user's message. However, the constructive interference techniques in MISO downlink channels exploit these cross correlations, which raise the question about their relation with multicast techniques.

Theorem 1: The optimal precoder for CIDC

$$
\begin{aligned}
& \mathbf{w}_{C I D C}(\mathbf{d}, \mathbf{H})=\arg \min _{\mathbf{w}} \operatorname{tr}\left(\mathbf{w} \mathbf{w}^{H}\right) \\
& \text { s.t. } \quad \angle\left(\mathbf{h}_{j} \mathbf{w}\right)=\angle\left(d_{j}\right) \quad \forall j \in K \\
& \mathbf{h}_{j} \mathbf{w} \mathbf{w}^{H} \mathbf{h}_{j}^{H}=\zeta_{j} \quad \forall j \in K .(28)
\end{aligned}
$$

is given by $\mathbf{w}_{C M C}\left(d, \mathbf{A}\left(d_{j}\right) \mathbf{H}\right)$ in 20 , where $\mathbf{A}(d)$

$$
\mathbf{A}(j, k)=\left\{\begin{array}{l}
\exp \left(\left(\angle d-\angle d_{j}\right) i\right), \quad j=k \\
0, \quad j \neq k .
\end{array}\right.
$$

Proof: We assume that we have the following equivalent channel as

$$
\mathbf{H}_{e}=\mathbf{A} \mathbf{H}
$$

The power minimization can be rewritten by replacing $\mathbf{H}$ by its equivalent channel $\mathbf{H}_{e}$ in 20 as

$$
\begin{array}{cl}
\min _{\mathbf{w}} & \left(\mathbf{w}_{e}^{H} \mathbf{w}_{e}\right) \\
\text { s.t. } & \angle\left(\mathbf{h}_{e, j} \mathbf{w}\right)=\angle(d) \quad \forall j \in K \\
& \mathbf{h}_{e, j} \mathbf{w} \mathbf{w}^{H} \mathbf{h}_{e, j}^{H}=\zeta_{j} \quad \forall j \in K .
\end{array}
$$

where $\mathbf{h}_{e, j}$ is the $j^{t h}$ row of the $\mathbf{H}_{e}$. The optimal precoder $\mathbf{w}_{e}$ for the equivalent channel can be expressed as

$$
\mathbf{w}_{e}=\sum_{j=1}^{K} \nu_{e, j} \mathbf{h}_{e, j}^{H} \text {. }
$$

Rewriting the first constraints in (31) as

$$
\begin{gathered}
\\
\angle\left(d-d_{j}\right) \angle\left(\mathbf{h}_{j} \mathbf{w}\right)=\angle(d) \\
\equiv \quad \angle\left(\mathbf{h}_{j} \mathbf{w}\right)=\angle\left(d_{j}\right) \quad \forall j \in K
\end{gathered}
$$

shows the equivalence between the constrained constellation multicast channel and constructive interference downlink channel.

Corollary 2: $K$ different M-PSK symbols can be received correctly at $K$ different users by using a single precoding vector $\mathbf{w} \in \mathbb{C}^{n_{t} \times 1}$, designed according to Theorem 1 , at the BS if $K \leq n_{t}$.

\section{NumericAl RESUlTS}

The channel between the base station and $j^{t h}$ user terminal is characterized by $\mathbf{h}_{j}=\sqrt{\gamma_{\circ}} \mathbf{h}_{j}^{\prime}$ where $\mathbf{h}_{j}^{\prime} \sim \mathcal{C N}(0,1)$, and $\gamma_{\circ}$ is the average channel power. In order to compare all described techniques, we used an energy efficiency metric as following

$$
\eta=\frac{\sum_{j} R_{j}}{P_{t o t}}
$$

where $R_{j}$ is the rate achieved by the $j^{\text {th }}$ user and given by $\log _{2}\left(1+\zeta_{j}\right), \zeta_{j}$ is selected to satisfy the related MPSK modulation and $P_{t o t}$ is the transmitted power by BS 


$$
\begin{array}{ccc}
0.5\left\|\mathbf{h}_{1}\right\|\left(\sum_{k}\left(-\mu_{k}+\alpha_{k} i\right)\left\|\mathbf{h}_{k}\right\| \rho_{1 k}\right. & - & \left.\sum_{k}\left(-\mu_{k}+\alpha_{k} i\right)\left\|\mathbf{h}_{k}\right\| \rho_{1 k}^{*}\right)=\sqrt{\zeta_{1}} \mathcal{I}(d) \\
0.5\left\|\mathbf{h}_{1}\right\|\left(\sum_{k}\left(-\mu_{k} i-\alpha_{k}\right)\left\|\mathbf{h}_{k}\right\| \rho_{1 k}\right. & + & \left.\sum_{k}\left(-\mu_{k} i-\alpha_{k}\right)\left\|\mathbf{h}_{k}\right\| \rho_{1 k}^{*}\right)=\sqrt{\zeta_{1}} \mathcal{R}(d) \\
& \vdots & \\
0.5\left\|\mathbf{h}_{K}\right\|\left(\sum_{k}\left(-\mu_{k}+\alpha_{k} i\right)\left\|\mathbf{h}_{k}\right\| \rho_{K k}\right. & - & \left.\sum_{k}\left(-\mu_{k}+\alpha_{k} i\right)\left\|\mathbf{h}_{k}\right\| \rho_{K k}^{*}\right)=\sqrt{\zeta_{K}} \mathcal{I}(d) \\
0.5\left\|\mathbf{h}_{K}\right\|\left(\sum_{k}\left(-\mu_{k} i-\alpha_{k}\right)\left\|\mathbf{h}_{k}\right\| \rho_{K k}\right. & + & \left.\sum_{k}\left(-\mu_{k} i-\alpha_{k}\right)\left\|\mathbf{h}_{k}\right\| \rho_{K k}^{*}\right)=\sqrt{\zeta_{K}} \mathcal{R}(d)
\end{array}
$$

$\operatorname{tr}\left(\mathbf{W} \mathbf{W}^{H}\right)$. The motivation of using this metric is the fact that CRZF and CIMRT do not aim at minimizing the transmitted power to grant certain quality of service by their design. Therefore, in some instance one or more users are given rate which is higher than the target rate. However for the multicast related techniques, the optimality holds when the target rate can be achieved exactly. For the sake of fairness in comparison, we use the metric in 34.

It can be seen in the Fig. (1) that energy efficiency for multicast with optimal input outperforms all the proposed techniques which confirms the fact of its optimality. For the downlink scheme derived from the constrained multicast takes the second place. It is shown that CRZF has the worst performance at all SNR values. However, its peer CIMRT has a superior performance on the expense of higher complexity due to the need for solving a non-linear set of equations.

Fig. (2) depicts the comparison between the optimal multicast

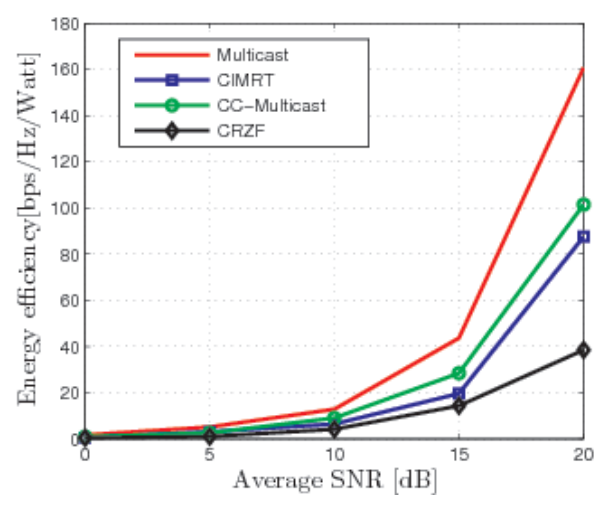

Fig. 1. Energy efficiency vs average SNR.

technique and constrained constellation multicast transmission from the power minimization perspective. The considered average SNR is $10 d B$, and all users have the same target rate. It can be concluded that the optimal multicast outperforms the constrained constellation multicast for all target rates, due to the fact that the constrained constellation multicast requires the phase alignment to ensure the correct reception of the target symbols.

Fig. (3) depicts the comparison between different techniques from energy efficiency perspective with increasing the target rates. It is clearly illustrated that CIMRT, constrained multicast and optimal multicast have very close performance at high target rates. Moreover, it can be concluded that CRZF has inferior performance with respect to the other techniques.

\section{CONCLUSIONS}

In this paper, we utilized jointly CSI and DI in symbol based precoding to exploit received interfering signal as use-

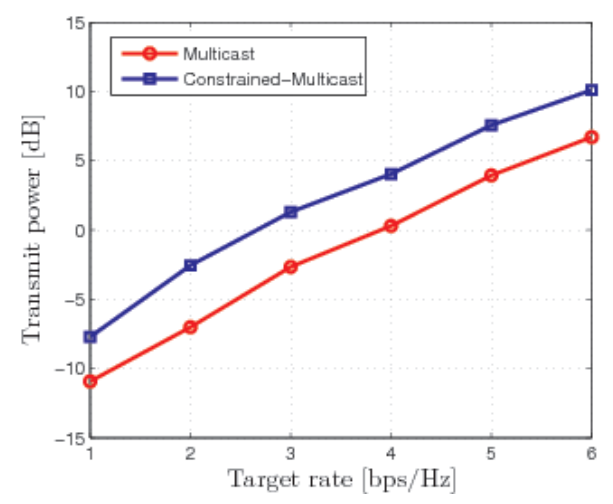

Fig. 2. Transmit power vs target rate.

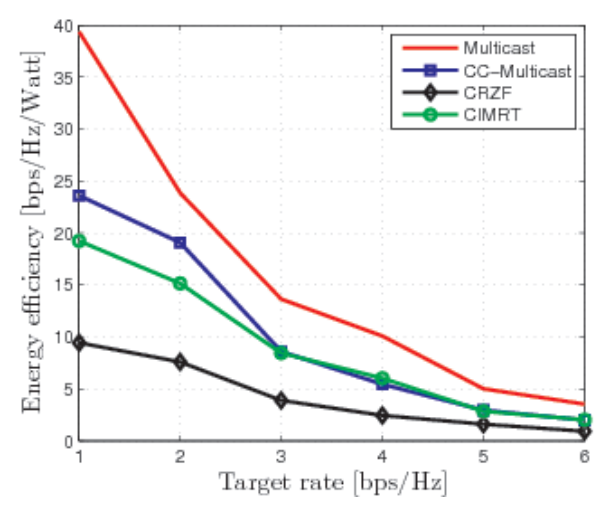

Fig. 3. Energy efficiency vs target rate

ful energy in constructive interference precoding. In these cases, the precoding design exploits the overlap in users' subspace instead of mitigating it. Therefore, we proposed a new technique based on MRT to constructively correlate the interference to enure the correct reception of data symbols. This fact enabled us finding the connection between the constructive interference precoding and multicast precoding wherein no interference should be mitigated. Therefore, we found the solution for power minimization considering two inputs scenario: the optimal input and the constrained constellation. From their closed formulations, we concluded that their transmissions should span the subspaces of each user. From the M-PSK constrained constellation multicast, we managed to find that the optimal constructive interference precoding can be expressed by solving this multicast problem assuming an equivalent channel.

\section{REFERENCES}

[1] N. D. Sidropoulos, T. N. Davidson, ans Z.-Q. Luo,"Transmit Beamforming for Physical-Layer Multicasting," IEEE Transactions on Signal Processing, vol. 54, no. 6, pp. 2239-2251, June 2006. 
[2] G. Caire, and S. Shamai (Shitz), "On the Achievable Throughput of a Multiantenna Gaussian Broadcast Channel," IEEE Transactions on Information Theory vol. 49, no. 7, pp. 1691 - 1706, July 2002.

[3] N. Jindal and Z.-Q. Luo, "Capacity Limits of Multiple Antenna Multicast," IEEE International Symposium on Information Theory (ISIT), pp. 1841 - 1845, June 2006.

[4] B. Du, M. Chen, W. Zhang and C. Pan,"Optimal beamforming for single group multicast systems based on weighted sum rate," IEEE International Conference on Communications(ICC), pp. 4921- 4925, June 2013.

[5] E. Jorswieck, "Beamforming in Interference Networks: Multicast, MISO IFC and Secrecy Capacity," International Zurich Seminars (IZS), March 2011.

[6] C. Masouros and E. Alsusa,"Dynamic Linear Precoding for the exploitation of Known Interference in MIMO Broadcast Systems," IEEE Transactions On Communications, vol. 8, no. 3, pp. 1396 - 1404, March 2009.

[7] C. Masouros, "Correlation Rotation Linear Precoding for MIMO Broadcast Communications," IEEE Transactions on Signal Processing, vol. 59, no. 1, pp. 252 262, January 2011.

[8] M. Alodeh, S. Chatzinotas, B. Ottersten, "Constructive Multiuser Interference in Symbol Level Precoding for the MISO Downlink Channel," IEEE Transactions on Signal Processing, vol. 63 , no. 9 , pp. 2239-2252, May 2015 